MPP-2010-15

LAPTH-004/10

\title{
Electroweak corrections to $W^{+} W^{-} Z$ and $Z Z Z$ production at the linear collider
}

\author{
Fawzi Boudjema, Sun Hao \\ LAPTH, Université de Savoie, CNRS, \\ BP 110, F-74941 Annecy-le-Vieux Cedex, France \\ Le Duc Ninh 1 , Marcus M. Weber \\ Max-Planck-Institut für Physik (Werner-Heisenberg-Institut), \\ D-80805 München, Germany
}

\begin{abstract}
We calculate the electroweak corrections to the production of $W^{+} W^{-} Z$ and $Z Z Z$ at the linear collider in the Standard Model. These processes are important for the extraction of the quartic couplings of the massive gauge bosons which can be a window on the mechanism of spontaneous symmetry breaking. We find that the weak corrections to some kinematic distributions show new features and hence cannot be explained by an overall scale factor.
\end{abstract}

The 9th Hellenic School and Workshops on Elementary Particle Physics and Gravity, August 31 - September 6, 2009, Corfu, Greece

\footnotetext{
${ }^{1}$ Speaker.
} 


\section{Introduction}

Due to its clean environment an $e^{+} e^{-}$linear collider in the $\mathrm{TeV}$ range is an ideal machine to probe in detail and with precision the inner working of the electroweak structure, in particular the mechansim of symmetry breaking. From this perspective the study of $e^{+} e^{-} \rightarrow W^{+} W^{-} Z$ and $e^{+} e^{-} \rightarrow Z Z Z$ may be very instructive and would play a role similar to $e^{+} e^{-} \rightarrow W^{+} W^{-}$at lower energies. Indeed it has been stressed that $e^{+} e^{-} \rightarrow$ $W^{+} W^{-} Z$ and $e^{+} e^{-} \rightarrow Z Z Z$ are prime processes for probing the quartic vector boson couplings [1]. In particular deviations from the gauge value in the quartic $W^{+} W^{-} Z Z$ and $Z Z Z Z$ couplings that are accessible in these reactions might be the residual effect of physics intimately related to electroweak symmetry breaking. Since these effects can be small and subtle, knowing these cross sections with high precision is mandatory. This calls for theoretical predictions taking into account loop corrections.

Radiative corrections to $e^{+} e^{-} \rightarrow Z Z Z$ have appeared recently in [2] and those to $e^{+} e^{-} \rightarrow W^{+} W^{-} Z$ in [3]. We have made an independent calculation of the electroweak corrections to $e^{+} e^{-} \rightarrow W^{+} W^{-} Z$ and $e^{+} e^{-} \rightarrow Z Z Z$, see [4]. Our preliminary results, eventually confirmed, have been presented in this workshop [5] prior to [3]. A detailed comparison between our results and the ones of Refs. [2, 3] has been done in [4].

In this report we summarize our results and make a further study on some distributions for $W^{+} W^{-} Z$ production.

\section{Calculational details}

Our calculations are done in the framework of the SM. At leading order the process $e^{+} e^{-} \rightarrow Z Z Z$ contains two types of couplings $e e Z$ and $Z Z H$. This process could probe the effect of a quartic $Z Z Z Z$ coupling which is absent at tree-level, in the SM. The process $e^{+} e^{-} \rightarrow W^{+} W^{-} Z$ is much more complicated with the involvement of trilinear and quartic gauge couplings in addition to the similar couplings as in $Z Z Z$ production. Compared to the well-tested process $e^{+} e^{-} \rightarrow W^{+} W^{-}$the new ingredients here are the two quartic gauge couplings $W W Z \gamma$ and $W W Z Z$. Thus, this $W W Z$ production at the ILC will be an excellent channel for studying these couplings.

We have performed the calculation in at least two independent ways both for the virtual and the real corrections leading to two independent numerical codes (one code is written in Fortran 77, the other in $\mathrm{C}++$ ). A comparison of both codes has shown full agreement at the level of the integrated cross sections as well as all the distributions that we have studied.

\section{Input parameters and renormalisation scheme:}

We follow closely the on-shell renormalisation scheme as detailed in Refs. [6, 7]. To make the final results independent of the light quark masses we adopt a variant of the $G_{\mu}$ scheme. At tree level, the electromagnetic coupling constant is calculated as $\alpha_{G_{\mu}}=\sqrt{2} G_{\mu} M_{W}^{2} s_{W}^{2} / \pi$. This absorbs some universal $m_{t}^{2}$ corrections and also the large logarithmic universal corrections proportional to $\ln \left(q^{2} / m_{f}^{2}\right)$ where $q$ is some typical energy scale and $m_{f}$ a fermion mass. To avoid double counting we have to subtract the one-loop part of the universal correction from the explicit $\mathcal{O}(\alpha)$ corrections by using the 
counterterm $\delta Z_{e}^{G_{\mu}}=\delta Z_{e}-\Delta r / 2$, the expression for $\Delta r$ can be found in [7]. For one-loop corrections we use the coupling $\alpha(0)$ for both virtual and real photons. Thus the NLO corrections are of order $\alpha_{G_{\mu}}^{3} \alpha(0)$. Further details and the complete set of input parameters are given in [4].

\section{Virtual corrections:}

The virtual corrections have been evaluated using a conventional Feynman-diagram based approach using standard techniques. We use the packages FeynArts and FormCalc-6.0 to generate all Feynman diagrams and helicity amplitude expressions [8]. We also use Sloops to check the correctness of the amplitudes by checking non-linear gauge invariance (see [9] and references therein). The total number of diagrams in the 't Hooft-Feynman gauge is about 2700 including 109 pentagon diagrams for $e^{+} e^{-} \rightarrow W^{+} W^{-} Z$ and about 1800 including 64 pentagons for $e^{+} e^{-} \rightarrow Z Z Z$. This already shows that $e^{+} e^{-} \rightarrow W^{+} W^{-} Z$ with as many as 109 pentagons is more challenging than $e^{+} e^{-} \rightarrow Z Z Z$. Indeed getting stable results for all scalar and tensor (up to rank 4) box integrals in the process $e^{+} e^{-} \rightarrow W^{+} W^{-} Z$ is a highly nontrivial task. An efficient way to solve this problem is by using higher precision arithmetic in part of the calculation. Further details related to loop integrals and the references for useful public codes are given in [4].

\section{Real corrections:}

In addition to the virtual corrections we also have to consider real photon emission, i.e. the processes $e^{+} e^{-} \rightarrow W^{+} W^{-} Z \gamma$ and $e^{+} e^{-} \rightarrow Z Z Z \gamma$. The corresponding amplitudes are divergent in the soft and collinear limits. The soft singularities cancel against the ones in the virtual corrections while the collinear singularities are regularized by the physical electron mass. To extract the singularities from the real corrections and combine them with the virtual contribution we apply both the dipole subtraction scheme and a phase space slicing method. The former is used to produce the final results since it yields smaller integration errors. Further details are given in [4].

\section{Defining the weak corrections:}

It is well-known that the collinear QED correction related to initial state radiation in $e^{+} e^{-}$-processes is large. In order to see the effect of the weak corrections, one should separate this large QED correction from the full result. It means that we can define the weak correction as an infrared and collinear finite quantity. The definition we adopt in this paper is based on the dipole subtraction formalism. In this approach, the sum of the virtual and the so-called "endpoint" (see [10] for the definition) contributions satisfies the above conditions and can be chosen as a definition for the weak correction

$$
\sigma_{\text {weak }}=\sigma_{\text {virt }}+\sigma_{\text {endpoint }} \text {. }
$$

For the numerical results shown in the next section, we will make use of this definition.

\section{Numerical results}

$\frac{e^{+} e^{-} \rightarrow Z Z Z:}{A}$

As shown in Fig. 1 the tree-level cross section rises sharply once the threshold for production opens, reaches a peak of about $1.1 \mathrm{fb}$ around a centre-of-mass energy of $600 \mathrm{GeV}$ before very slowly decreasing with a value of about $0.9 \mathrm{fb}$ at $1 \mathrm{TeV}$. The full NLO corrections are quite large and negative around threshold, $-35 \%$, decreasing sharply to stabilise 

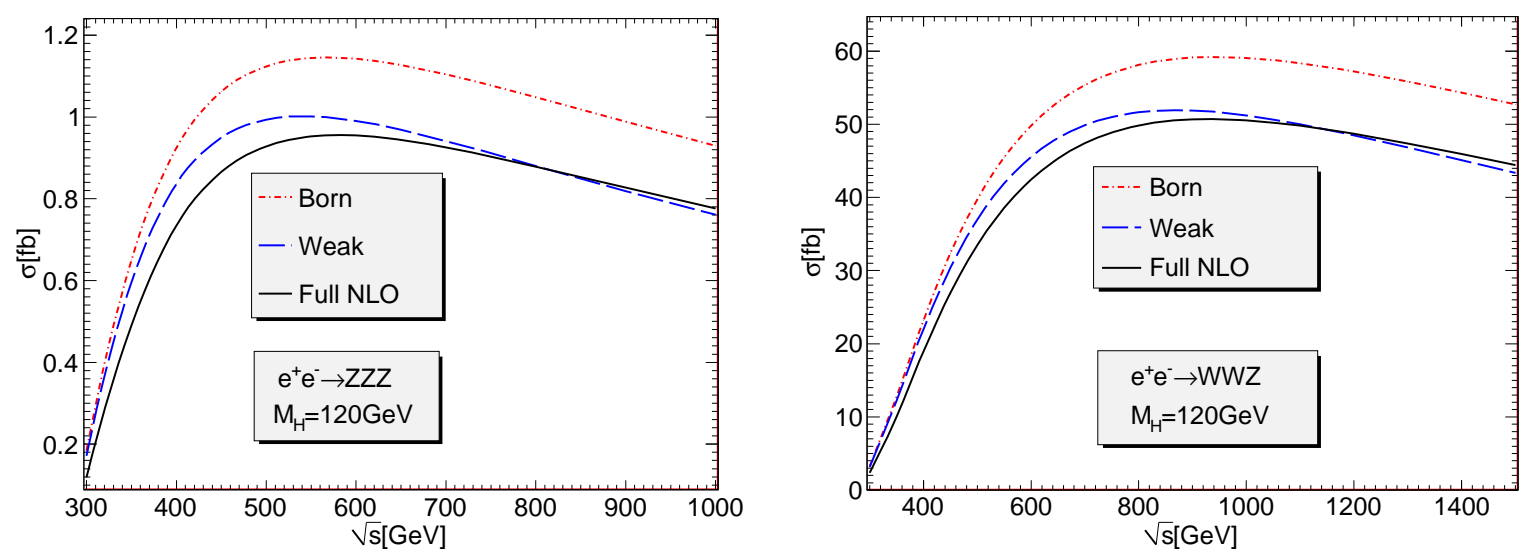

Figure 1: Left: the total cross section for $e^{+} e^{-} \rightarrow Z Z Z$ as a function of $\sqrt{s}$ for the Born, full $\mathcal{O}(\alpha)$ and genuine weak correction. Right: the same for $e^{+} e^{-} \rightarrow W^{+} W^{-} Z$.

at a plateau around $\sqrt{s}=600 \mathrm{GeV}$ with $-16 \%$ correction. The sharp rise and negative corrections at low energies are easily understood. They are essentially due to initial state radiation (ISR) and the behaviour of the tree-level cross section. The photon radiation reduces the effective centre-of-mass energy and therefore explains what is observed in the figure. On the other hand the genuine weak corrections, in the $G_{\mu}$ scheme, are relatively small at threshold, $-7 \%$. They however increase steadily with a correction as large as $-18 \%$ at $\sqrt{s}=1 \mathrm{TeV}$.

$\underline{e^{+} e^{-} \rightarrow W^{+} W^{-} Z:}$

Compared to $Z Z Z$ production, the cross section for $e^{+} e^{-} \rightarrow W^{+} W^{-} Z$ is almost 2 orders of magnitudes larger for the same centre-of-mass energy. For example at $500 \mathrm{GeV}$ it is about $40 \mathrm{fb}$ at tree level, compared to $1 \mathrm{fb}$ for the $e^{+} e^{-} \rightarrow Z Z Z$ cross section. For an anticipated luminosity of $1 \mathrm{ab}^{-1}$, this means that the cross section should be known at the per-mil level. The behaviour of the total cross section as a function of energy resembles that of $e^{+} e^{-} \rightarrow Z Z Z$. It rises sharply once the threshold for production opens, reaches a peak before very slowly decreasing as shown in Fig. 1. However as already discussed the value of the peak is much larger, $\sim 50 \mathrm{fb}$ at NLO, moreover the peak is reached around $\sqrt{s}=1 \mathrm{TeV}$, much higher than in $Z Z Z$. This explains the bulk of the NLO corrections at lower energies which are dominated by the QED correction, large and negative around threshold and smaller at higher energies. As the energy increases the weak corrections get larger reaching about $-18 \%$ at $\sqrt{s}=1.5 \mathrm{TeV}$.

In Fig. 2 we show the distributions in the rapidity and the transverse momentum of the $W^{+}$. First, due to photon radiation, in the full NLO corrections some large corrections do show up at the edges of phase space. However when the QED corrections are subtracted, the weak corrections cannot be parameterized by an overall scale factor, for all the distributions that we have studied. This feature together with possible normal/anomalous thresholds make it clear that calculating explicitly one-loop EW corrections is needed for a precise comparison with experimental data. 

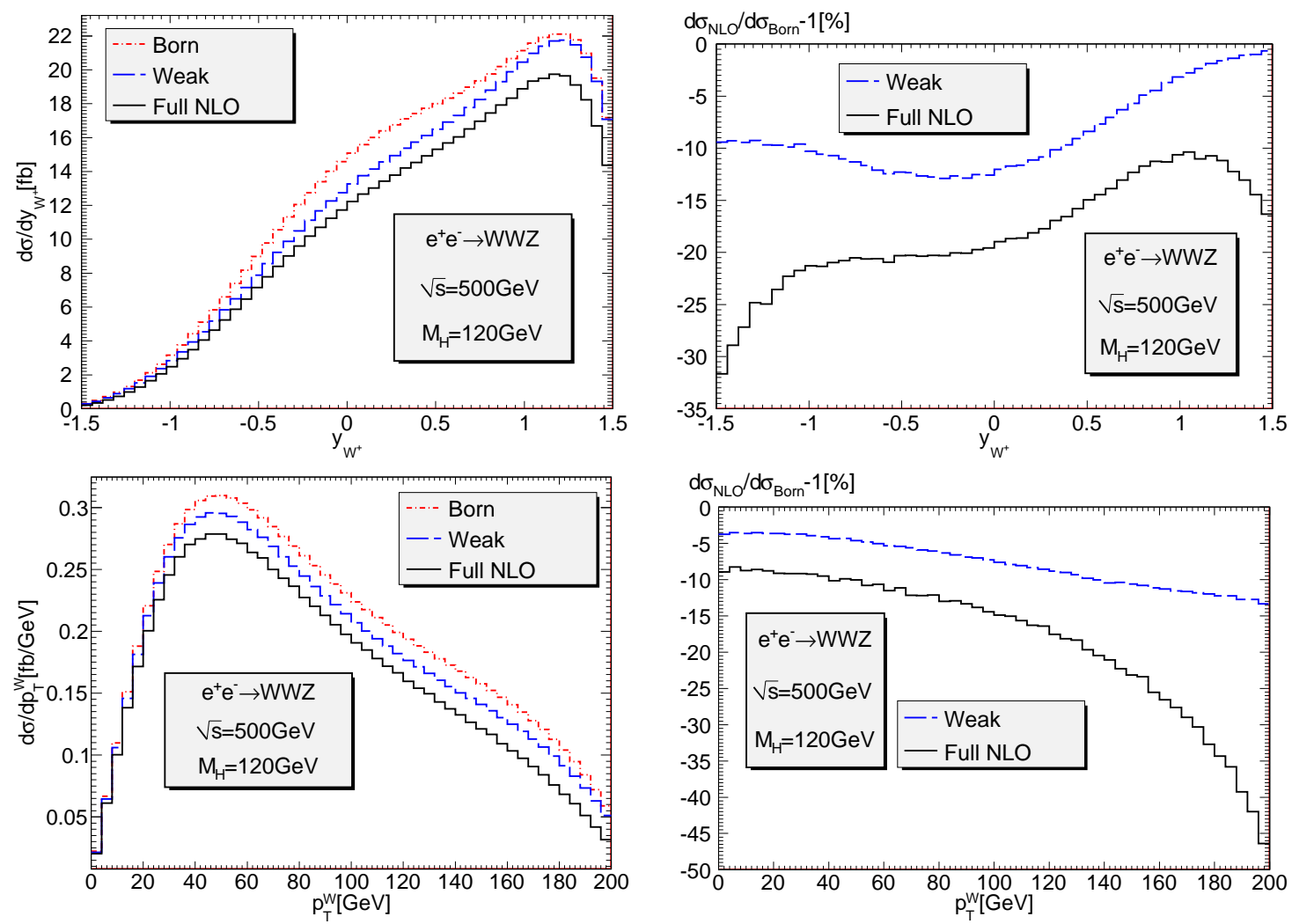

Figure 2: From top to bottom: distributions for the rapidity and the transverse momentum of the $W^{+}$for $e^{+} e^{-} \rightarrow W^{+} W^{-} Z$. The panels on the left show the tree-level, the full NLO and the weak correction. The panels on the right show the corresponding relative (to the tree-level) percentage corrections.

\section{References}

[1] G. Belanger and F. Boudjema, Phys. Lett. B288, 201 (1992).

[2] S. Ji-Juan et al., Phys. Rev. D78, 016007 (2008), arXiv:0807.0669.

[3] S. Wei et al., Phys. Lett. B680, 321 (2009), arXiv:0909.1064.

[4] F. Boudjema, L. D. Ninh, S. Hao, and M. M. Weber, arXiv:0912.4234.

[5] http://www.physics.ntua.gr/corfu2009/Talks/leducninh@gmail_com_01.pdf.

[6] G. Belanger et al., Phys. Rept. 430, 117 (2006), hep-ph/0308080.

[7] A. Denner, Fortschr. Phys. 41, 307 (1993), arXiv:0709.1075.

[8] T. Hahn, Comput. Phys. Commun. 140 (2001) 418, hep-ph/0012260;

T. Hahn, M. Perez-Victoria, Comput. Phys. Commun. 118 (1999) 153, hep-ph/9807565.

[9] N. Baro, F. Boudjema, and A. Semenov, Phys. Rev., D78 115003 (2008), arXiv:0807.4668.

[10] S. Dittmaier, Nucl. Phys. B565, 69 (2000), hep-ph/9904440. 